OPEN ACCESS

Edited by:

Eric Spierings,

Utrecht University, Netherlands

Reviewed by:

Marieke Griffioen,

Leiden University Medical Center, Netherlands

Yongxia Wu,

Medical University of South Carolina,

United States

${ }^{*}$ Correspondence:

Andreas Beilhack

beilhack_a@ukw.de

${ }^{\text {t}}$ These authors have contributed equally to this work

Specialty section:

This article was submitted to

Alloimmunity and Transplantation,

a section of the journal

Frontiers in Immunology

Received: 10 August 2020

Accepted: 11 December 2020

Published: 29 January 2021

Citation:

Bäuerlein CA, Qureischi M, Mokhtari Z, Tabares P, Brede C,

Jordán Garrote AL, Riedel SS,

Chopra M, Reu S, Mottok A,

Arellano-Viera E, Graf C, Kurzwart $M$,

Schmiedgen $K$, Einsele $H$, Wölfl $M$,

Schlegel PG and Beilhack $A$

(2021) A T-Cell Surface Marker

Panel Predicts Murine Acute

Graft-Versus-Host Disease.

Front. Immunol. 11:593321.

doi: 10.3389/fimmu.2020.593321

\section{A T-Cell Surface Marker Panel Predicts Murine Acute Graft-Versus-Host Disease}

\author{
Carina A. Bäuerlein ${ }^{1,2,3 \dagger}$, Musga Qureischi ${ }^{1,2,3 \dagger}$, Zeinab Mokhtari ${ }^{1,2}$, Paula Tabares ${ }^{1,2}$, \\ Christian Brede ${ }^{1,2,3}$, Ana-Laura Jordán Garrote ${ }^{1,2,3}$, Simone S. Riedel ${ }^{1,2,3}$, \\ Martin Chopra ${ }^{1,2}$, Simone Reu ${ }^{4}$, Anja Mottok ${ }^{4}$, Estibaliz Arellano-Viera ${ }^{1,2}$ ', Carolin Graf ${ }^{1,2}$, \\ Miriam Kurzwart ${ }^{1,2}$, Katharina Schmiedgen ${ }^{1,2}$, Hermann Einsele ${ }^{1,3}$, Matthias Wölff $\left.\right|^{3,5}$, \\ Paul-Gerhardt Schlegel ${ }^{3,5}$ and Andreas Beilhack ${ }^{1,2,3 *}$
}

\begin{abstract}
${ }^{1}$ Department of Medicine II, University Hospital of Würzburg, Würzburg, Germany, ${ }^{2}$ Interdisciplinary Center for Clinical Research (IZKF), Würzburg University, Würzburg, Germany, ${ }^{3}$ Graduate School of Life Sciences, Würzburg University, Würzburg, Germany, ${ }^{4}$ Institute of Pathology, Würzburg University, Würzburg, Germany, ${ }^{5}$ Department of Pediatrics, University Hospital of Würzburg, Würzburg, Germany
\end{abstract}

Acute graft-versus-host disease (aGvHD) is a severe and often life-threatening complication of allogeneic hematopoietic cell transplantation (allo-HCT). AGvHD is mediated by alloreactive donor T-cells targeting predominantly the gastrointestinal tract, liver, and skin. Recent work in mice and patients undergoing allo-HCT showed that alloreactive T-cells can be identified by the expression of $\alpha 4 \beta 7$ integrin on T-cells even before manifestation of an aGvHD. Here, we investigated whether the detection of a combination of the expression of $\mathrm{T}$-cell surface markers on peripheral blood (PB) CD8 ${ }^{+} \mathrm{T}$ cells would improve the ability to predict aGvHD. To this end, we employed two independent preclinical models of minor histocompatibility antigen mismatched alloHCT following myeloablative conditioning. Expression profiles of integrins, selectins, chemokine receptors, and activation markers of PB donor T-cells were measured with multiparameter flow cytometry at multiple time points before the onset of clinical aGvHD symptoms. In both allo-HCT models, we demonstrated a significant upregulation of $\alpha 4 \beta 7$ integrin, CD162E, CD162P, and conversely, a downregulation of CD62 L on donor T-cells, which could be correlated with the development of aGvHD. Other surface markers, such as CD25, CD69, and CC-chemokine receptors were not found to be predictive markers. Based on these preclinical data from mouse models, we propose a surface marker panel on peripheral blood T-cells after allo-HCT combining $\alpha 4 \beta 7$ integrin with CD62L, CD162E, and CD162P (cutaneous lymphocyte antigens, CLA, in humans) to identify patients at risk for developing aGvHD early after allo-HCT.

\footnotetext{
Keywords: acute graft-versus-host disease, alloreactive T cells, transplantation, prediction, mouse models
} 


\section{INTRODUCTION}

Allogeneic hematopoietic cell transplantation (allo-HCT) has proven as a curative therapy for life-threatening diseases of the hematopoietic system such as leukemia and lymphomas $(1,2)$. However, acute graft-versus-host disease (aGvHD) remains the leading cause of death of transplant-related complications resulting in up to $20 \%$ mortality after allo-HCT $(3,4)$. As early prediction of aGvHD remains difficult, potent preemptive immunosuppressive regimens have been established that require swift adaptation as soon as first clinical aGvHD symptoms appear. Intensifying immunosuppression early, however, puts patients at high risk of infectious complications, whereas late treatment often comes too late to avoid disease exacerbation. Considering the occurrence of $\mathrm{aGvHD}$ as an end-result of a forceful and highly dynamic immune response may explain how difficult it is to treat patients once aGvHD occurs. Preclinical studies in animal models have revealed a pathophysiological cascade of events that precede aGvHD onset. First, host conditioning triggers responses to tissue injury such as neoangiogenesis (5), recruitment of innate immune cells, such as neutrophilic granulocytes and monocytes (6-8), and release of cytokines and cytokine receptors (9). Concomitantly, naïve donor T-cells migrate to secondary lymphoid organs (SLOs), undergo allo-antigen priming, proliferate, differentiate into effector T-cells, and migrate from these priming sites to peripheral tissues such as the intestinal tract, liver, and skin, where they cause organ damage resulting in clinical aGvHD manifestation (10-13).

Thus, to improve clinical outcome it had become clear that identifying and monitoring predictive markers to identify patients at risk of developing clinically relevant aGvHD are warranted and these endeavors are starting to bear fruits (1416). Tissue damage, release of damage-associated factors and cytokines can be picked up by proteomic and systems biology analysis approaches to guide treatment decisions and provide a basis for specific therapeutic interventions (17).

Recent insights into aGvHD pathophysiology supported by observations in mouse models uncovered a temporally and spatially defined aGvHD initiation and effector phase $(11,13$, 18). Extensive studies of the initiation and effector phases of alloreactive $\mathrm{T}$-cells have revealed that after priming of alloreactive T-cells in SLOs, donor T-cells are mobilized into the peripheral blood (PB) before homing to target tissues of aGvHD. In minor histocompatibility antigen mismatch (miHAg) allo-HCT mouse models the transition from initiation of aGvHD to the aGvHD effector phase can last for an extended period of two weeks before the first clinical symptoms of aGvHD occur (19). In this transition phase alloreactive T-cells are circulating in the PB before entering target organs. This phenomenon can also be observed in patients before the onset of clinically apparent aGvHD (20). The timely homing of alloreactive donor T-cells to aGvHD target tissues depends on the combination of the appropriate surface receptor expression profile (21). We hypothesized that these homing receptors could provide a unique footprint to distinguish alloreactive T-cells in the PB. As distinct surface receptors have been functionally implicated in the development of aGvHD, the close monitoring of PB T-cells and their unique surface marker profile might be an appropriate approach to predict the risk of aGvHD even before the release of tissue damage associated surface markers. Indeed, Paszesny and colleagues identified a $\mathrm{CD}^{+} \mathrm{CD} 146^{+} \mathrm{CCR}^{+} \mathrm{T}$-cell population in the peripheral blood before the onset of aGvHD that predicted patient outcome (22).

Here, we investigated surface receptors on $\mathrm{PB} \mathrm{CD} 8^{+} \mathrm{T}$-cells in two independent mouse models after miHAg allo-HCT before aGvHD symptoms appear. We identified a combination of homing receptors, namely $\alpha 4 \beta 7$ integrin, CD162E, CD162P, and CD62L, on $\mathrm{PB} \mathrm{CD} 8^{+} \mathrm{T}$-cells that may serve as an early potential biomarker panel to predict onset of aGvHD. Surprisingly, CC-chemokine receptors deemed not suitable to identify alloreactive $\mathrm{CD} 8^{+} \mathrm{T}$-cells. Our observations provide the basis to prospectively evaluate corresponding surface receptors on T-cells in patients undergoing allo-HCT to unambiguously identify a surge of alloreactive T-cells before clinical symptoms occur.

\section{MATERIALS AND METHODS}

\section{Mice}

BALB/b (H-2 $\left.{ }^{\mathrm{b}}, \mathrm{CD} 90.2\right)$, B10.D2 (H-2 $\left.{ }^{\mathrm{d}}, \mathrm{CD} 90.2\right)$, and congenic $\mathrm{C} 57 \mathrm{Bl} / 6\left(\mathrm{~B} 6, \mathrm{H}-2^{\mathrm{b}}, \mathrm{CD} 90.1\right)$ mice were obtained from Jackson Laboratories (Bar Harbor, ME), C57Bl/6 (B6, H-2 $\left.2^{\mathrm{b}}, \mathrm{CD} 90.2\right)$, and $\mathrm{BALB} / \mathrm{c}\left(\mathrm{H}-2^{\mathrm{d}}, \mathrm{CD} 90.2\right)$ mice were obtained from Charles River (Sulzfeld, Germany). All mice were housed in a pathogen-free facility at the Center for Experimental Molecular Medicine (ZEMM), Würzburg. All experiments were performed according to the German regulations for animal experimentation and governmental approval (Permit 55.2-2531.01-30/09, 55.2-2531.0182/14, and 55.2.2-2532-2-537-22).

\section{Allogeneic and Syngeneic HCT}

8 - to 12-week-old female recipient mice were myeloablatively irradiated (BALB/b, BALB/c, B10.D2 8 Gy; B6 9 Gy) with an electron linear accelerator (Mevatron Primus, Siemens, Germany) prior to HCT. For hematopoietic reconstitution $\mathrm{B} 6 \rightarrow \mathrm{BALB} / \mathrm{b}$ allo-HCT recipients (or syngeneic $\mathrm{B} 6$ controls) were injected intravenously with $5 \times 10^{6}$ WT B6 bone marrow (BM) cells and $5 \times 10^{6}$ splenic $\mathrm{CD}^{+}$and $\mathrm{CD}^{+} \mathrm{B} 6 \mathrm{~T}$-cells (expressing the congeneic marker CD90.1). Myeloablatively conditioned B10.D2 $\rightarrow \mathrm{BALB} / \mathrm{c}$ allo-HCT recipients (or syngeneic B10.D2 controls) were transplanted with $5 \times 10^{6} \mathrm{BM}$ cells and $3 \times 10^{7}$ splenocytes (containing an equivalent of $1 \times 10^{7}$ $\mathrm{CD}^{+}$and $\mathrm{CD}^{+}$donor T-cells without congeneic marker). Bone marrow controls received $5 \times 10^{6} \mathrm{BM}$ cells only. Transplanted mice were monitored daily for survival, weight change, and clinical aGvHD symptoms according to Cooke et al (23).

\section{Histopathological Analysis}

Mice were euthanized on day +25 after HCT and organs were fixed in $4 \%$ PFA (Paraformaldehyde) in PBS. Tissues were cut into $2 \mu \mathrm{m}$ sections and stained with $\mathrm{H} \& \mathrm{E}$ (hematoxylin and eosin). GvHD scoring was performed according to Lerner et al. (24). Healthy tissue 
was considered as 0 , whereas mild and moderate tissue damage were graded with 1-2. Severe tissue damage was considered as 3. All samples were graded by unbiased pathologists in a blinded fashion.

\section{Flow Cytometry}

Peripheral blood samples of murine HCT recipients and untreated controls were collected at indicated time points. Erythrocytes were lysed and after washing cells were blocked with normal rat serum and stained with appropriate antibodies at $4^{\circ} \mathrm{C}$ for $30 \mathrm{~min}$. The following anti-mouse antibodies (clones) were used: anti-CD3e (17A2), anti-CD8 $\alpha$ (53-6.7), anti-CD4 (RM4-5), anti-CD45.1

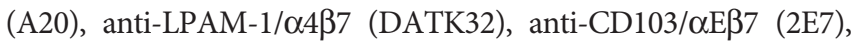
anti-CCR4 (2G12), anti-CCR5 (C34-3448), anti-CCR7 (4B12), anti-CD25 (PC61), anti-CD44 (IM7), anti-CD62L (MEL-14), antiCD69 (H1.2F3) (Biolegend, Uithoorn, Netherlands); anti-CD90.1 (H1S51) (eBioscience, Frankfurt, Germany); anti-CCR2 (475301), anti-CCR6 (FAB590P), anti-CCR9 (242503), E-selectin ligand-Fc chimera (R\&D Systems, Germany), P-selectin ligand-IgG fusion protein (BD, Heidelberg, Germany); anti-human-IgG-FITC (Jackson ImmunoResearch Laboratories, West Grove, PA). Dead cells were excluded with propidium iodide (PI, Invitrogen, Darmstadt, Germany) or Zombie Aqua ${ }^{\mathrm{TM}}$ Fixable Viability Kit staining (Biolegend). Flow cytometry was performed on a FACS Canto II (BD), and data was analyzed with FlowJo (BD) and Infinicyt Software (Cytognos, Salamanca, Spain). Gates were set using the fluorescence-minus-one gating strategy. Anti-mouse or anti-rat/hamster CompBeads (BD) were used for compensation.

\section{Statistical Analyses, Principal Component Analysis and K-Means Data Clustering}

Repeated measurements are expressed as the mean \pm standard deviation. To compare miHAg mismatch versus syngeneic recipients for several time points, a repeated measures ANOVA was conducted with a posthoc analysis using a Bonferroni correction of the significance level. Data was analyzed with GraphPad Prism 9 software (La Jolla, CA, USA) and IBM SPSS 22 (Armonk, NY, USA). Data reaching statistical significance is indicated as ${ }^{\star} P \leq 0.05,{ }^{*} P \leq 0.01,{ }^{* *} P \leq 0.001$.

To extract the variance of a data set, multivariate principal component analysis (PCA) was employed. Data dimensionality was reduced while retaining the original data information. Each sample was displayed by a data point in a $2 \mathrm{D}$ principal component space (PC1 and PC2) and data points with similar characteristics were grouped together while the different data groups were visualized in PC space.

Unsupervised K-means clustering was employed to partition the unlabeled data set into K clusters where the mean distances between data points in the same cluster was minimized.

\section{RESULTS}

\section{Onset of aGvHD in Two miHAg Mismatch Allo-HCT Mouse Models}

GvHD target organ infiltration depends on the appropriate expression of homing receptors on migrating cells (21).
Therefore, we tested an extended panel of surface markers consisting of adhesion molecules, chemokine receptors, and activation markers to determine potential biomarkers that may specifically define alloreactive T-cells in the $\mathrm{PB}$. To this end, we compared two $\mathrm{CD}^{+}$and $\mathrm{CD}^{+}$T-cell dependent miHAg mismatched allogeneic mouse models $(\mathrm{B} 6 \rightarrow \mathrm{BALB} / \mathrm{b}$ and B10.D2 $\rightarrow$ BALB/C, Figure 1A) with syngeneic HCT recipients and healthy WT controls. Based on our previous results of donor T-cell migration kinetics (19), we chose two peak time points during the early and later phase of T-cell mobilization to the $\mathrm{PB}$ (days+6 and +15) and included day +21 (clinically apparent aGvHD onset after murine miHAg allo-HCT) as appropriate for a precise receptor analysis.

First, we analyzed whether miHAg B10.D2 $\rightarrow$ BALB/c alloHCT showed the same aGvHD kinetics as the previously reported $\mathrm{B} 6 \rightarrow \mathrm{BALB} / \mathrm{b}$ model (19). Host conditioning regimen and transplantation procedure resulted in $20 \%-30 \%$ weight loss during the first 8 days after allo-HCT in B10.D2 $\rightarrow$ BALB/c miHAg mismatched recipients of $\mathrm{BM}$ and T-cells. Mice recovered after 10 days and reached $80 \%-90 \%$ of their initial body weight but could not recover completely. Weight remained at the same level until the end of the experiment. In contrast, weight loss of syngeneic B10.D2 $\rightarrow$ B10.D2 recipients was rather mild during the first week after HCT (10\%) compared to BM recipients (15\%-20\%). Both groups recovered 10 days after HCT and reached their starting weight (Figure 1B). In allo-HCT recipients, typical signs of aGvHD occurred around day+20. Symptoms included diarrhea, skin inflammation, and ruffled fur (Figure 1C).

Histopathological analysis confirmed aGvHD in the small and large bowel as well as the skin on day+25 after B10.D2 $\rightarrow$ BALB/C allo-HCT (Figures 1D, E). Increased tissue damage, T-cell infiltration, and crypt apoptosis contributed to the significant aGvHD histological score in the large bowel whereas typical signs of aGvHD in the skin featured necrotic keratinocytes, inflammation, and basal vacuolization of the epidermis.

\section{Alloreactive Donor CD8 ${ }^{+}$T-Cells Upregulate Homing Molecules Early After HCT}

Next, we collected blood samples of the tail vein from allogeneic and syngeneic recipients on days $+6,+15$, and +21 for immune phenotyping of $\mathrm{PB} \mathrm{CD} 8^{+} \mathrm{T}$-cells (gating strategy for the $\mathrm{B} 6 \rightarrow \mathrm{BALB} / \mathrm{b}$ model is shown in Supplementary Figure 1) and compared these to the respective untreated controls (Figure 2).

$\mathrm{PB} \mathrm{CD} 8^{+}$T-cells highly up-regulated the mucosal homing receptor $\alpha 4 \beta 7$ integrin after miHAg mismatch allo-HCT (Figure 2A, representative histograms) in both miHAg models. In the allogeneic $\mathrm{B} 6 \rightarrow \mathrm{BALB} / \mathrm{b}$ model, $\alpha 4 \beta 7^{+} \mathrm{CD}^{+} \mathrm{T}$-cell frequencies in the $\mathrm{PB}$ significantly exceeded those after syngeneic HCT at all time points and the expression peaked at day+15 $\left(28.7 \pm 3.9 \%\right.$ vs. $8.1 \pm 2.1 \%, P<0.0001 ;<1 \% \alpha 4 \beta 7^{+} \mathrm{PB}$ $\mathrm{CD}^{+}$T-cells in untreated controls, Figure 2B, Supplementary Figure 2). Similarly, $\mathrm{PB} \mathrm{CD} 8^{+} \mathrm{T}$-cells of $\mathrm{B} 10 . \mathrm{D} 2 \rightarrow \mathrm{BALB} / \mathrm{c}$ miHAg mismatch recipients expressed significantly higher levels of $\alpha 4 \beta 7$ 
A
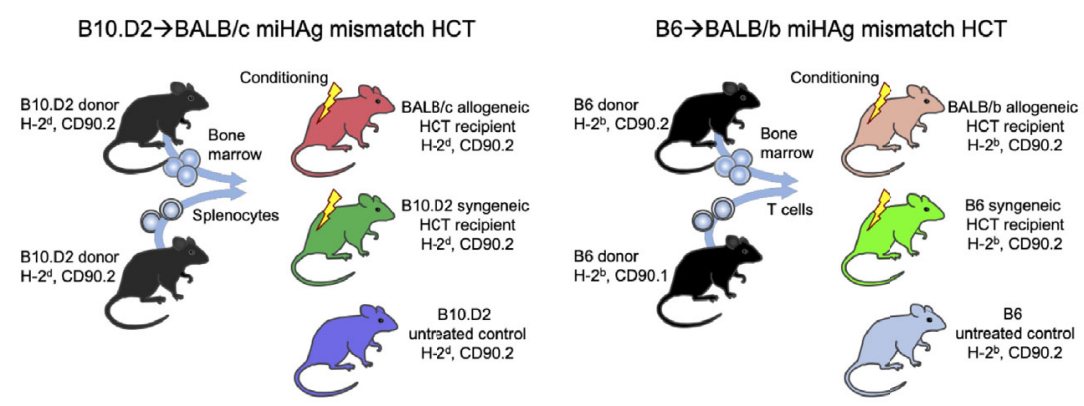

B

C
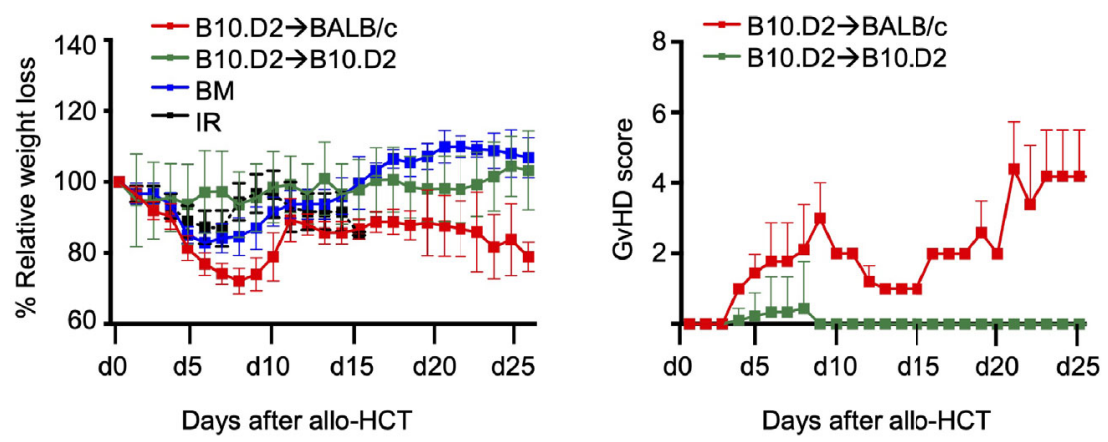

D

E
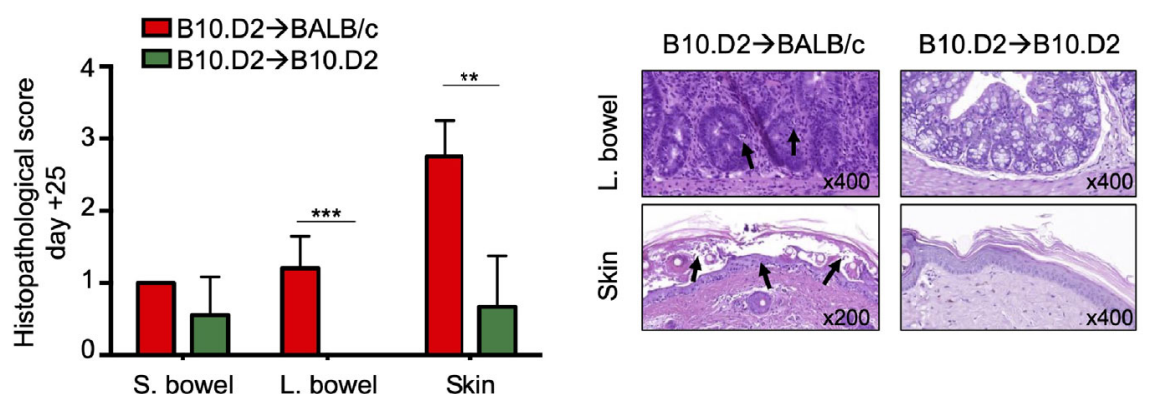

FIGURE 1 | Murine MHC matched miHAg mismatched allo-HCT models to assess a biomarker panel on peripheral blood (PB) CD8 ${ }^{+}$T-cells for aGvHD. (A) Schematic overview of the two described miHAg mismatch allo-HCT and syngeneic HCT models. (B-E) Validation of aGvHD induction in the B10.D2 $\rightarrow$ BALB/C miHAg mismatch allo-HCT model. (B) Weight loss relative to day 0 of HCT of the B10.D2 $\rightarrow$ BALB/c model and the respective control groups. (C) Clinical scores during aGvHD progression of miHAg mismatch and syngeneic recipients. (D) Histopathological grading of the aGvHD target organs intestine and skin on day +25 after allo-HCT and (E) representative hematoxylin-eosin stains at indicated magnification. Values are displayed as means \pm SD. MiHAg mismatch B10.D2 $\rightarrow$ B10.D2 $\mathrm{BM}+\mathrm{T}$-cells, $\mathrm{n}=$ 9; syngeneic B10.D2 $\rightarrow$ B10.D2 BM+T-cells, $\mathrm{n}=9$; BM only, $\mathrm{n}=5$; irradiation only, $\mathrm{n}=5$. $T=\mathrm{T}$-cells, $\mathrm{BM}=$ Bone Marrow, IR = Irradiation.

integrin compared to syngeneic controls at all measured time points and $\alpha 4 \beta 7$ expression peaked on day+15 (42.72 $\pm 5.3 \%$ vs. $2.4 \pm 0.8 \%, P=0.0002$, Figure $2 \mathrm{C}$ ). Next, we analyzed expression levels of the skin homing receptors CD162E and CD162P after allo-HCT. Notably, in B10.D2 $\rightarrow$ BALB/c miHAg allo-HCT recipients, $\mathrm{CD} 162 \mathrm{E}$ expression levels of alloreactive $\mathrm{PB} C \mathrm{CD}{ }^{+} \mathrm{T}-$ cells exceeded significantly the levels found in syngeneic controls at all measured time points (e.g., $74.3 \pm 11.2 \%$ vs. $28.1 \pm 6.95 \%$ on day $+6, P<0.0001$ ) (Figures $2 \mathrm{~A}, \mathrm{C}$ ). On day +6 , in $\mathrm{B} 6 \rightarrow \mathrm{BALB} / \mathrm{b}$ miHAg allo-HCT recipients, $\mathrm{CD} 162 \mathrm{E}^{+} \mathrm{CD}^{+} \mathrm{T}$-cell frequencies did not differ between allogeneic and syngeneic HCT recipients (Figure 2B). However, thereafter, levels of CD162 $\mathrm{E}^{+} \mathrm{T}$-cells after
miHAg mismatch allo-HCT exceeded those found in syngeneic controls $(20.5 \pm 4.4 \%$ versus $8.5 \pm 4.7 \%$ on day+ $15, \mathrm{p}=0.1)$. By day+21, CD162E expression levels almost equaled between both groups again. In contrast, in untreated controls less than $5 \%$ of $\mathrm{PB}$ $\mathrm{CD}^{+}$T-cells expressed CD162E under steady-state conditions.

Additionally, $\mathrm{PB} \mathrm{CD}^{+}$T-cells highly up-regulated CD162P in both miHAg allo-HCT models (Figures 2B, C) compared to syngeneic controls. In $\mathrm{B} 6 \rightarrow \mathrm{BALB} / \mathrm{b} \mathrm{miHAg}$ allo-HCT recipients, $45.2 \pm 4.4 \%$ of $\mathrm{CD}^{+}{ }^{+} \mathrm{T}$-cells expressed CD162P already on day +6 compared to $13.8 \pm 6.8 \%$ of $\mathrm{CD}^{+}{ }^{+} \mathrm{T}$-cells in syngeneic controls $(P=0.01)$ and less than $10 \%$ of $\mathrm{CD}^{+} \mathrm{T}$-cells in untreated controls. Already by day+6 in B10.D2 $\rightarrow \mathrm{BALB} / \mathrm{c}$ allo-HCT, 
A
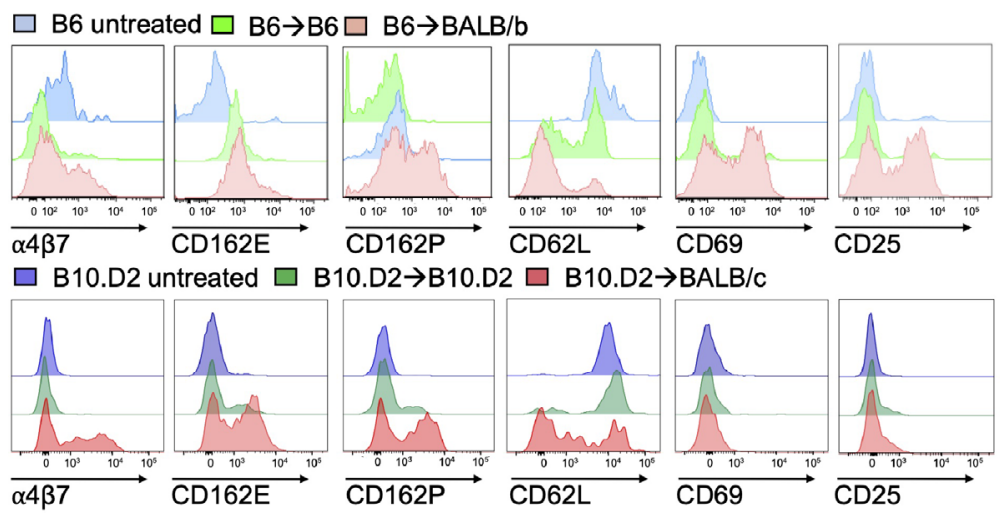

B

$\mathrm{B} 6 \rightarrow \mathrm{BALB} / \mathrm{b} \square \mathrm{B} 6 \rightarrow \mathrm{B} 6 \quad \square \mathrm{B} 6$ untreated
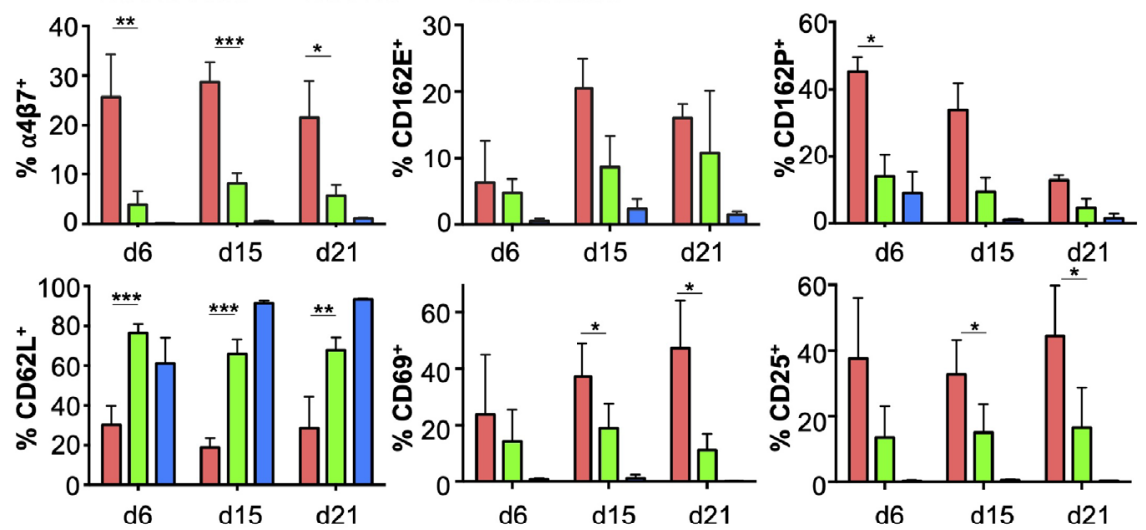

\section{C}
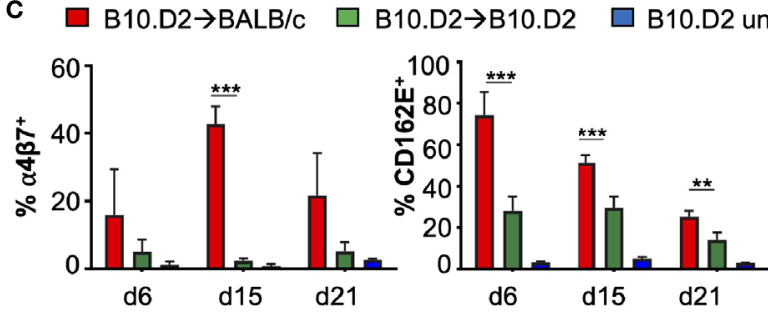

untreated
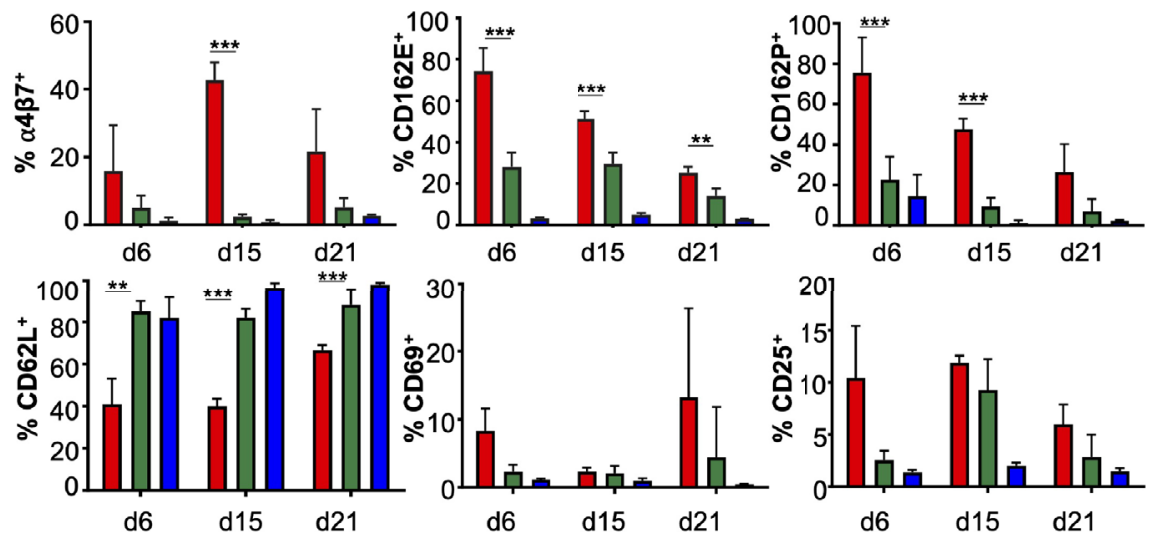

FIGURE 2 | Allogeneic peripheral blood CD8 ${ }^{+}$T-cells display a distinct surface marker profile before the onset of aGvHD. (A) Representative histograms show surface receptor expression on PB CD8 ${ }^{+}$T-cells on day +15 (upper row B6 $\rightarrow$ BALB/B model, lower row B10.D2 $\rightarrow$ BALB/C model) after allo-HCT compared to the respective expression levels of syngeneic and untreated healthy controls. (B, C) Summary of surface receptor expression on $\mathrm{PB}$ CD8 ${ }^{+} \mathrm{T}$-cells at indicated time points after HCT and untreated controls. Values are displayed as means \pm SD. C57BI/6 $\rightarrow$ BALB/b: $n=3-6$ in each group, B10.D2 $\rightarrow$ BALB/C: $n=5-9$ in miHAg mismatch and syngeneic control group, untreated B10.D2 $n=4$. Statistical significance between miHAg mismatch recipients and syngeneic controls was determined with a repeated ANOVA and posthoc analysis using Bonferroni correction of the significance level. ${ }^{\star} P \leq 0.05 ;{ }^{* \star} P \leq 0.01 ;{ }^{\star \star \star} P \leq 0.001$.

$\mathrm{CD}^{+} \mathrm{CD} 162 \mathrm{P}^{+}$expression levels of allo-HCT recipients significantly exceeded those of syngeneic recipients (75.6 \pm $17.4 \%$ vs $22.6 \pm 11.4 \% ; P=0.0001)$.

Furthermore, the lymphoid homing receptor CD62L significantly decreased on PB CD8 ${ }^{+}$T-cells after allo-HCT and remained significantly lower compared to syngeneic HCT at all analyzed time points $(30.2 \pm 9.6 \%$ vs. $76.4 \pm 4.4 \%, P=<0.0001$ on day +6 after B $6 \rightarrow$ BALB/b allo-HCT and $41 \pm 12.3 \%$ vs. $85.2 \pm$ $5 \%, P=0.0019$ on day+6 after B10.D2 $\rightarrow$ BALB/c allo-HCT, respectively). In syngeneic recipients, $\mathrm{PB} \mathrm{CD}^{+} \mathrm{CD}^{2} 2 \mathrm{~L}^{+} \mathrm{T}$-cell 
frequencies were only slightly lower than those of untreated controls (75 to $95 \% \mathrm{CD}_{2} 2 \mathrm{~L}^{+}$).

To assess the activation status of $\mathrm{PB} \mathrm{CD} 8^{+} \mathrm{T}$-cells we investigated the expression of CD69 and CD25. Frequencies of PB CD $8^{+} \mathrm{CD} 69^{+}$ T-cells differed significantly between the two groups only on day+15 $(37.1 \pm 11.8 \%$ in allogeneic $\mathrm{B} 6 \rightarrow \mathrm{BALB} / \mathrm{b}$ vs. $18.9 \pm 8.7 \%$ in syngeneic recipients, $P=0.0409 ;<3 \%$ of circulating $\mathrm{CD} 8^{+} \mathrm{CD} 69^{+}$ T-cells in untreated controls) and +21 (Figure 2B). After $\mathrm{B} 10 . \mathrm{D} 2 \rightarrow \mathrm{BALB} / \mathrm{c}$ allo-HCT, CD69 expression exceeded the levels measured on $\mathrm{PB} \mathrm{CD8} 8^{+} \mathrm{T}$-cells of syngeneic controls on day+6 (8.3 \pm 3.2 vs. $2.3 \pm 1.1 ; P=0.08$, representative histograms in Figure 2A).

$\mathrm{PB} \mathrm{CD} 8^{+} \mathrm{T}$-cells of $\mathrm{B} 6 \rightarrow \mathrm{BALB} / \mathrm{b}$ allo-HCT recipients significantly up-regulated the activation marker CD25 compared to syngeneic controls on days +15 and day $+21(32.7 \pm 10.5 \%$ $\mathrm{CD}^{+} \mathrm{CD} 25^{+} \mathrm{T}$-cells in allogeneic vs. $15.03 \pm 8.7 \%$ in syngeneic recipients, $P=0.03$, Figure $2 \mathbf{B}$ ). Yet, no significant differences between allogeneic and syngeneic recipients were determined after B10.D2 $\rightarrow$ BALB/c allo-HCT (Figure 2C).

Other markers such as CC-chemokine receptors (CCRs 2, 4, $5,6,7,9)$ surprisingly never differed between allogeneic and syngeneic recipients (Supplementary Figure 3). At day+6, PB $\mathrm{CD}^{+}$T-cells highly up-regulated all CCRs both in allogeneic $\mathrm{B} 6 \rightarrow \mathrm{BALB} / \mathrm{b}$ recipients and in syngeneic $\mathrm{B} 6 \rightarrow \mathrm{B} 6$ controls compared to untreated animals. After day+6, CCR expression levels decreased similarly in allogeneic and syngeneic recipients. The differences in CCR expression levels did not reach statistical significance at any of the analyzed time points.

\section{Combined Surface Marker Panel Predicts aGvHD Onset}

Based on these results, we next asked whether the combination of T-cell surface markers can clearly discriminate allogeneic transplanted mice from syngeneic recipients and untreated controls (Figure 3A). Our principle component analysis considered the four surface markers $\alpha 4 \beta 7$ integrin, CD162E, CD162P, and CD62L as CD25, CD69, and CC-chemokine receptors were not exclusively expressed on alloreactive T-cells. To classify the animals based on their treatment, we applied an unsupervised $\mathrm{K}$-means clustering on the percentage of $\mathrm{CD} 8^{+} \mathrm{T}$ cells with high expression levels of the four aforementioned surface markers for day +6 as an early GvHD prediction would be most beneficial. We compared the clustering based on four markers to a clustering based on the single marker $\alpha 4 \beta 7$ integrin to investigate if the combination of several markers could enhance the predictive value (Figure 3B). The first two principal component spaces (PC1 and $\mathrm{PC} 2$ ) predict the variance in the data. The clustering analysis based on $\alpha 4 \beta 7$ integrin alone could unequivocally separate allogeneic from syngeneic recipients on day +15 but not on day +6 . Determining the accuracy score [= (number of true classified samples)/ (number of total test data)] for several marker combinations on day+6 showed that in both models the maximum prediction accuracy could be obtained with a minimum of three parameters (Figure 3C). Conclusively, our data suggest that the early upregulation of $\alpha 4 \beta 7$ integrin, CD162E, CD162P as well as low expression levels of $\mathrm{CD} 62 \mathrm{~L}$ on circulating $\mathrm{PB} \mathrm{CD}^{+} \mathrm{T}$-cells serve as strong predictor of $\mathrm{aGvHD}$ and that the combination of at least three markers increases the precision of our analysis.

\section{DISCUSSION}

In this study, we present a combinatorial panel of $\alpha 4 \beta 7$ integrin, CD162E, CD162P, and CD62L as predictive to unequivocally identify alloreactive $\mathrm{CD}^{+} \mathrm{T}$-cells in the $\mathrm{PB}$ of murine allo-HCT recipients (Figure 4). Benefiting from insights into donor T-cell migration kinetics in the PB early after HCT (19), we analyzed the dynamic changes of surface markers on donor $\mathrm{CD}^{+} \mathrm{T}$-cells after miHAg mismatch allo-HCT. The combination of these markers proved superior to single markers especially at early time points after allo-HCT allowing a reliable prediction for early time points. Examining if the combination of the expression of chemokine receptors, homing and activation markers could give more defined insights into T-cell homing to specific target tissues of aGvHD we confirmed in two independent miHAg allo-HCT models that the expression of $\alpha 4 \beta 7$ integrin, CD162E, CD162P, and lack of CD62L on $\mathrm{PB} \mathrm{CD}^{+} \mathrm{T}$-cells preceded the onset of aGvHD. Conversely, we could exclude an extended panel of surface receptors (several chemokine receptors, CD69, and CD25) as these did not turn out as reliable predictors.

\section{Are These Identified Markers Functionally Linked to aGvHD Pathophysiology?}

Our analysis in two independent murine models of aGvHD, confirmed previous studies in mice showing that alloreactive $\mathrm{CD}^{+}$donor T-cells highly up-regulate $\alpha 4 \beta 7$ integrin at early time points after HCT (25-27). In patients, upregulation of $\alpha 4 \beta 7$ integrin on conventional T-cells or reduction of $\alpha 4 \beta 7^{+}$Tregs, respectively, correlated with intestinal aGvHD manifestation (28-30). Accordingly, $\alpha 4 \beta 7$ integrin also proved as a relevant therapeutic target for the treatment of intestinal inflammation. Vedolizumab, a monoclonal antibody against $\alpha 4 \beta 7$, is approved for ulcerative colitis and under current investigation for the preemptive therapy of aGvHD (NCT03657160, ClinicalTrials.gov). The frequency of $\alpha 4 \beta 7^{+} \mathrm{T}$-cells in the $\mathrm{PB}$ of colitis ulcerosa patients before Vedolizumab treatment could predict treatment outcome (31). Therefore, $\alpha 4 \beta 7$ integrin not only appears as an attractive diagnostic and prognostic biomarker for intestinal aGvHD but may also be predictive for treatment outcome.

In both murine miHAg allo-HCT models, allogeneic circulating $\mathrm{PB} \mathrm{CD}^{+}$T-cells upregulated the skin homing receptors $\mathrm{CD} 162 \mathrm{E}$ and $\mathrm{CD} 162 \mathrm{P}$. Both mouse surface molecules (CD162E, CD162P) expressed by allogeneic donor T-cells or the corresponding human skin homing receptor CLA, respectively, are strongly associated with aGvHD initiation $(32,33)$, even if redundancies with other homing pathways have been observed (34). Patients suffering from aGvHD showed higher levels of circulating $\mathrm{CLA}^{+} \mathrm{T}$-cells compared to patients who did not develop aGvHD (35). 

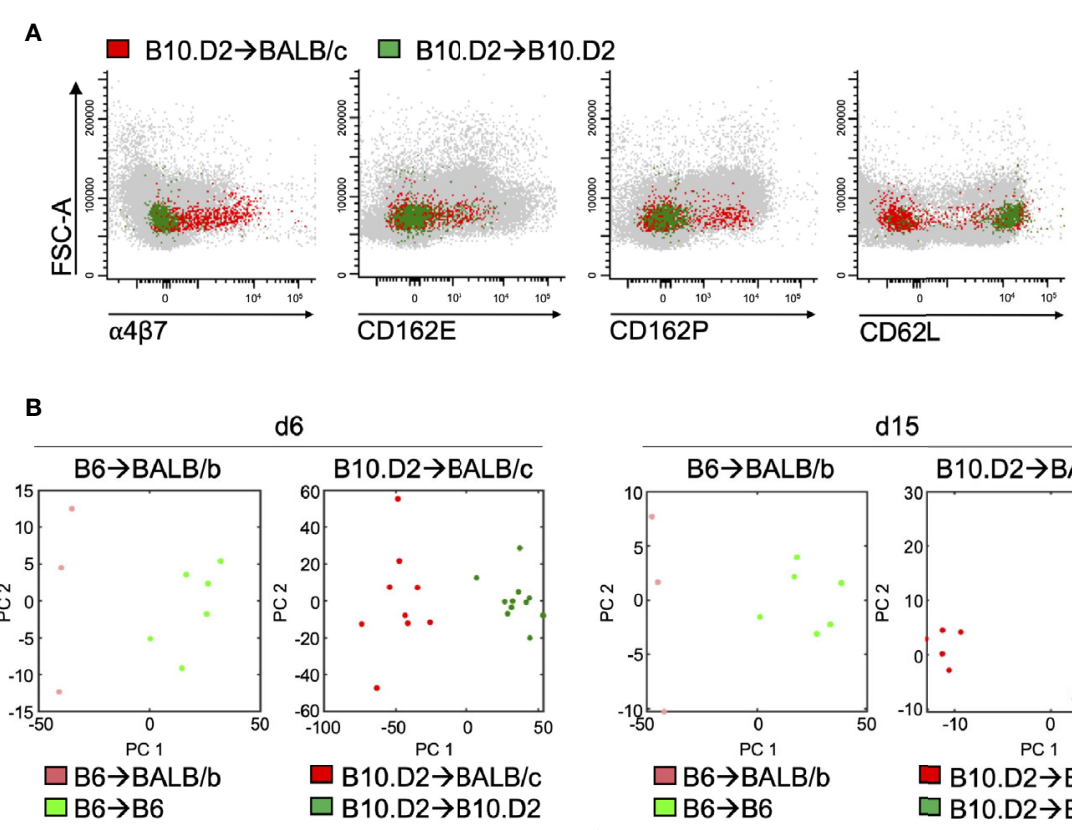

d6
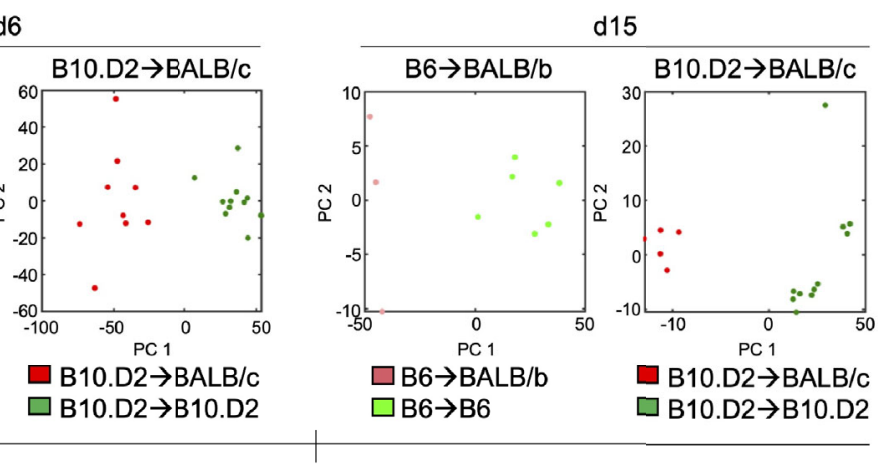

Parameters: $\alpha 4 \beta 7$, CD162E, CD162P, CD62L

C

$\mathrm{B} 6 \rightarrow \mathrm{BALB} / \mathrm{b} \quad \square \mathrm{B} 10 . \mathrm{D} 2 \rightarrow \mathrm{BALB} / \mathrm{C}$

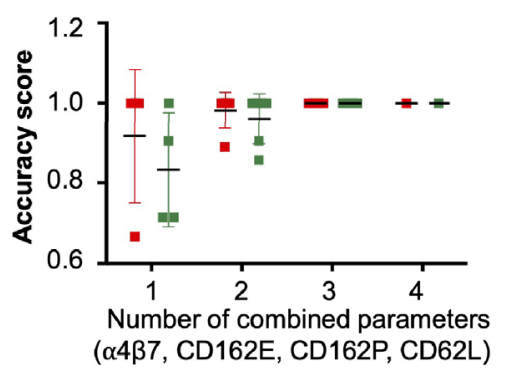

FIGURE 3 | Combination of surface receptors defines alloreactive T-cells in the PB of allo-HCT recipient. (A) Representative FACS plots of PB samples on day+15 analyzed with Infinicyte Flow Cytometry Software. Overlay of allogeneic (red) vs. syngeneic (green) $\mathrm{CD}^{+} \mathrm{CD} 8^{+} \mathrm{T}$-cells of total peripheral blood mononuclear cells (PBMC, in grey). (B) Principal component analysis based on four parameters and unsupervised K-means clustering of individual samples. Each data point represents one mouse. Red dots show allogeneic and green dots show syngeneic recipients of both models. Distances between data points represent the similarity.

(C) Determination of accuracy score [= (number of true classified samples)/(number of total test data)] for the combination of parameters. Each point represents one possible marker combination.

The lymphoid homing receptor CD62L is expressed on naïve, central memory T-cells but also on T-cells with a stem-cell like memory phenotype (36). Enhanced mobilization of $\mathrm{CD} 8^{+} \mathrm{CD} 44^{\mathrm{hi}} \mathrm{CD} 62 \mathrm{~L}^{\mathrm{low}}$ effector T-cells and a reduced number of even circulating $\mathrm{CD} 2 \mathrm{~L}^{+}$naïve T-cells were demonstrated to be a strong indicator of aGvHD days and in our hands even two weeks before the onset of aGvHD.

However, as a cautionary note in the interpretation of our presented data, we have to point out that in both independent miHAg allo-HCT GvHD models, mice developed intestinal, liver and skin aGvHD. Therefore, the proposed panel may have prognostic value that there is an increased risk of aGvHD development, but at this stage of our study it is too early to draw conclusions about the specific organ manifestation.

\section{Are More T-Cell Biomarkers Better Than One?}

In our unbiased clustering analysis, $\alpha 4 \beta 7$ integrin alone could not robustly separate allogeneic from syngeneic recipients on day+6 after allo-HCT two weeks before clinical aGvHD onset, whereas the combination of surface markers proved highly reliable $[\alpha 4 \beta 7, \mathrm{CD} 162 \mathrm{E} / \mathrm{CD} 162 \mathrm{P}$ (CLA) and CD62L]. By determination of the accuracy parameter we observed the maximum precision of our predictive test when we combined 


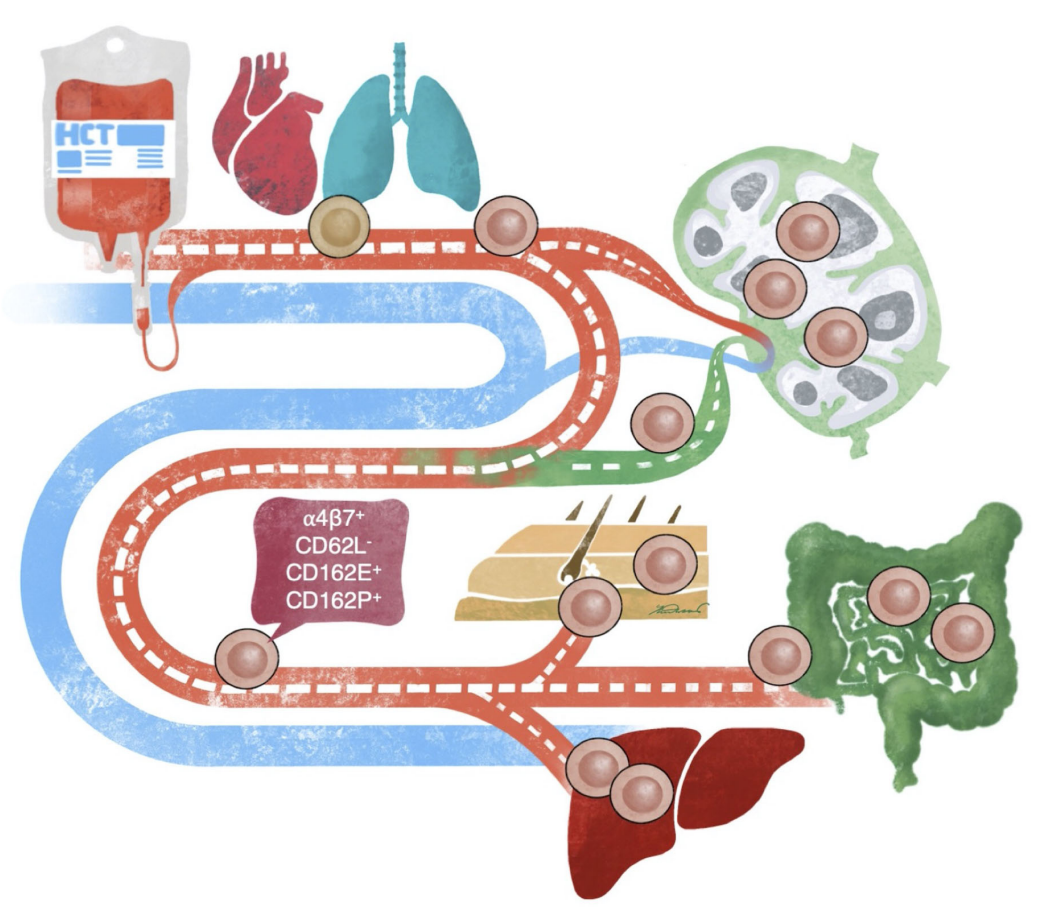

FIGURE 4 | Proposed biomarkers to measure circulating alloreactive CD8 ${ }^{+}$T-cells before aGvHD onset. Based on our results we propose a panel of $\alpha 4 \beta 7$ integrin, CD62L, CD162E, and CD162P CD8 ${ }^{+}$T-cell surface markers to predict onset of aGvHD as early as day +6 after allo-HCT. The proposed marker panel relies on data from two independent $\mathrm{CD} 4^{+}$and $\mathrm{CD} 8^{+} \mathrm{T}$-cell driven miHAg allo-HCT mouse models. Notably, T-cell reconstitution after allo-HCT can take four weeks or even longer. However, upon antigen contact T-cell mediated alloimmune responses take only a few days to be unleashed allowing for the discrimination of alloreactive vs. reconstituting T-cell populations. For translation of our findings into clinical studies it will be important to factor in the heterogeneity of the patient population in terms of patient history, conditioning regimens, genetics, and immunosuppressive GvHD prophylaxis.

three markers. However, we have observed already a high accuracy score $(0.87 \pm 0.15)$ for only one single parameter, which might be explained by our well-controlled experimental setting and the relatively low sample size. Increasing the sample size and keeping in mind the heterogeneity of patient samples, we propose to increase the number of measured parameters to a minimum of three parameters.

As the accuracy score for three combined markers reached already the maximum and did not change by adding another marker in both models, it is tempting to limit future testing to a panel of three surface markers on $\mathrm{PB} \mathrm{CD} 8^{+} \mathrm{T}$ cells. However at this stage, as pointed out above, the panel cannot predict sitespecific aGvHD manifestation yet. As in the clinical scenarios, one can expect a much higher heterogeneity in patient samples, we would recommend to consider more than three markers for an exploratory patient study, even if a stringent consideration of three markers may turn out sufficient as in our preclinical mouse models to predict aGvHD onset. In summary, based on our results, we expect that a combined marker panel of at least three markers will prove valuable for an early prediction of aGvHD in allograft recipients.

\section{What Are the Limitations of This Study?}

First, while our results were highly reproducible in two independent mouse models, the situation in human patients undergoing allo-HCT is certainly more complex (37). Both miHAg allo-HCT mouse models and syngeneic controls relied on a myeloablative irradiation only-based conditioning regimen, whereas in patients a variety of conditioning protocols are used. Second, genetic uniformity of donors and recipients utilizing inbred mouse strains contrast to the enormous genetic heterogeneity in patients who also vary in their clinical history, age, underlying diseases, co-morbidities, and previous medications. Third, mice were housed in homogenous specific pathogen-free microbial conditions and did not receive any immunosuppressive GvHD prophylaxis. Steroids can have an impact on the expression levels of homing receptors (38). Furthermore, by design of our study, mice did not suffer from malignant diseases or succumbed to infections. Future studies will have to address whether the panel of $\mathrm{T}$ cell markers allows discrimination between allo-HCT patients at risk for aGvHD and patients suffering from opportunistic infections or both, e.g., increased risk for aGvHD development and/or cytomegalovirus (CMV)-reactivation. Biomarkers for the beneficial graft-versusleukemia response and the efficient combat of opportunistic infections still need to be defined. However, a reductionist approach employing miHAg GvHD mouse models has the advantage to evaluate the reproducibility of candidate biomarkers. By utilizing one miHAg allo-HCT model for a discovery set of biomarkers and the second miHAg allo-HCT 
model for validation, these models helped to identify a panel of biomarkers that now need to be rigorously tested in patients while ruling out a range of preconceived candidates.

In contrast to the analyzed integrin and selectin family members, none of the tested CC-chemokine receptors could give more insights into T-cell homing to different tissues or GvHD onset. This may be partially explained by their dynamic regulation, vulnerability to loss of expression and artifacts upon ex vivo cell manipulation and redundant expression of CCRs (39). Yet, inflammation after conditioning appears to trigger CCR and endothelial ligand expression (40,41), consistent with our observed CCR up-regulation in both allogeneic and syngeneic recipients. Of note, over time CCR expression decreased, suggesting that host conditioning upregulated CCRs on CD8 T-cells independent of the allogeneic or syngeneic setting. Nevertheless, CCRs still hold the potential as therapeutic targets. For instance, Reshef et al. reported the beneficial effects of CCR5 blockade, which resulted in reduced aGvHD in phase I/II clinical trials $(42,43)$. These effects were consistent with previously published results in murine models $(44,45)$.

\section{How Can These Findings Be Translated to the Clinics?}

Unbiased clustering analysis revealed a panel of four $\mathrm{CD}^{+} \mathrm{T}$-cell surface markers ( $\alpha 4 \beta 7$, CD162E/CD162P (CLA), CD62L) in two independent miHAg allo-HCT models. This suggests that flow cytometric analysis of PB T-cells also in patients undergoing alloHCT could predict aGvHD before patients become symptomatic. Based on these results, we recommend to prospectively test the combination of the aforementioned surface molecules in alloHCT patients at early time points. Cytotoxic $\mathrm{CD}^{+}$effector Tcells are key players in aGvHD pathophysiology. Thus, it appears attractive to design GvHD prophylaxis protocols based on the detection of circulating alloreactive T-cells, even before they can exert their tissue destructive force. As time to onset of aGvHD markedly varies between patients, it will be important to design a prospective clinical trial based on a calendar-driven collection to standardize data acquisition plus an event-driven sample collection. Second, as efficient homing of immune effector cells is a prerequisite to combat infections and to exert the desired GvL effect, a vigorous prospective study will require a relatively large sample size and meticulous clinical and laboratory documentation to discern aGvHD causing alloimmune responses from these scenarios. Furthermore, it will be interesting to compare and cross-validate the proposed panel of four surface flow cytometric biomarkers with currently investigated serum and urine biomarkers (46-48). Assessment of T-cell surface markers in real-time will help to bring down GvHD-related mortality by early recognition and intervention.

\section{REFERENCES}

1. Jenq RR, van den Brink MRM. Allogeneic haematopoietic stem cell transplantation: individualized stem cell and immune therapy of cancer. Nat Rev Cancer (2010) 10(3):212-21. doi: 10.1038/nrc2804

\section{DATA AVAILABILITY STATEMENT}

The original contributions presented in the study are included in the article/Supplementary Material. Further inquiries can be directed to the corresponding author.

\section{ETHICS STATEMENT}

All experiments were performed according to the German regulations for animal experimentation and governmental approval (Permit 55.2-2531.01-30/09, 55.2-2531.01-82/14 and 55.2.2-2532-2-537-22).

\section{AUTHOR CONTRIBUTIONS}

$\mathrm{CB}$ and MQ performed and CB, A-LJG, SSR, MC, EA-V, CG, $\mathrm{MK}, \mathrm{KS}$, and MW helped with experiments. SR and AM performed histopathologic analyses. CB, MQ, ZM, MW, and $\mathrm{AB}$ analyzed data, designed research, and wrote the paper. P-GS and HE helped write the paper. All authors contributed to the article and approved the submitted version.

\section{FUNDING}

This study was supported by a Deutsche José Carreras LeukämieStiftung (DJCLS F 11/04) research fellowship to CB, grants of the Wilhelm-Sander-Stiftung (2007-081), Interdisciplinary Center for Clinical Research Würzburg (IZKF, B-233) and the Deutsche Forschungsgemeinschaft DFG SFB TR221 (Project number 324392634: B09, Z02 and A03), the Europäische Fonds für Regionale Entwicklung (EFRE) and an unrestricted donation by Merete and Alexander Knauf to AB. MQ was awarded with a $\mathrm{PhD}$ fellowship from the Graduate School of Life Sciences Würzburg of the German Excellence Initiative.

\section{ACKNOWLEDGMENTS}

We thank Dr. Franz Schwab for expert technical assistance and the members of the Beilhack lab for helpful discussions.

\section{SUPPLEMENTARY MATERIAL}

The Supplementary Material for this article can be found online at: https://www.frontiersin.org/articles/10.3389/fimmu.2020. 593321/full\#supplementary-material

2. Norkin M, Wingard JR. Recent advances in hematopoietic stem cell transplantation. F1000Research (2017) 6(0):1-7. doi: 10.12688/ f1000research.11233.1

3. Zeiser R, Blazar B. Acute Graft-versus-Host Disease - Biologic Process, Prevention, and Therapy. N Engl J Med (2017) 377(22):2167-79. doi: 10.1056/NEJMra1609337 
4. Chow EJ, Cushing-Haugen KL, Cheng GS, Boeckh M, Khera N, Lee SJ, et al. Morbidity and mortality differences between hematopoietic cell transplantation survivors and other cancer survivors. J Clin Oncol (2017) 35 (3):306-13. doi: 10.1200/JCO.2016.68.8457

5. Riesner K, Shi Y, Jacobi A, Kräter M, Kalupa M, McGearey A, et al. Initiation of acute graft-versus-host disease by angiogenesis. Blood (2017) 129 (14):2021-32. doi: 10.1182/blood-2016-08-736314

6. Socié G, Mary JY, Lemann M, Daneshpouy M, Guardiola P, Meignin V, et al. Prognostic value of apoptotic cells and infiltrating neutrophils in graft-versushost disease of the gastrointestinal tract in humans: TNF and Fas expression. Blood (2004) 103(1):50-7. doi: 10.1182/blood-2003-03-0909

7. Schwab L, Goroncy L, Palaniyandi S, Gautam S, Triantafyllopoulou A, Mocsai A, et al. Neutrophil granulocytes recruited upon translocation of intestinal bacteria enhance graft-versus-host disease via tissue damage. Nat Med (2014) 20(6):64854. doi: $10.1038 / \mathrm{nm} .3517$

8. Klämbt V, Wohlfeil SA, Schwab L, Hülsdünker J, Ayata K, Apostolova P, et al. A Novel Function for P2Y2 in Myeloid Recipient-Derived Cells during Graftversus-Host Disease. J Immunol (2015) 195(12):5795-804. doi: 10.4049/ jimmunol.1501357

9. Piper C, Drobyski WR. Inflammatory cytokine networks in gastrointestinal tract graft vs. Host disease. Front Immunol (2019) 10(2):1-10. doi: 10.3389/ fimmu.2019.00163

10. Korngold R, Sprent J. Negative selection of T cells causing lethal graft-versushost disease across minor histocompatibility barriers. Role of the $\mathrm{H}-2$ complex. J Exp Med (1980) 151(5):1114-24. doi: 10.1084/jem.151.5.1114

11. Beilhack A, Schulz S, Baker J, Beilhack GF, Wieland CB, Herman EI, et al. In vivo analyses of early events in acute graft-versus-host disease reveal sequential infiltration of T-cell subsets. Blood (2005) 106(3):1113-22. doi: 10.1182/blood-2005-02-0509

12. El-Asady R, Yuan R, Liu K, Wang D, Ronald E, Philip JL, et al. TGF- $\beta$ dependent CD103 expression by CD8+ T cells promotes selective destruction of the host intestinal epithelium during graft-versus-host disease. J Exp Med (2005) 201(10):1647-57. doi: 10.1084/jem.20041044

13. Panoskaltsis-Mortari A, Price A, Hermanson JR, Taras E, Lees C, Serody SJ, et al. In vivo imaging of graft-versus-host-disease in mice. Blood (2004) 103 (9):3590-8. doi: 10.1182/blood-2003-08-2827

14. Harris A, Ferrara J, Levine J. Advances in predicting acute GVHD. $\mathrm{Br} J$ Haematol (2013) 160(3):288-302. doi: 10.1111/bjh.12142

15. Etra A, Gergoudis S, Morales G, Kowalyk S, Lin J-Y, Shah J, et al. Comparison of Gvhd Biomarker Algorithms for Predicting Lethal Gvhd and Non-Relapse Mortality. Biol Blood Marrow Transplant (2019) 25(3):53-4. doi: 10.1016/ j.bbmt.2018.12.131

16. Rowan CM, Paczesny S. Biomarkers for Early Complications After Hematopoietic Stem Cell Transplantation. Clinics Lab Med (2019) 39 (1):61-72. doi: 10.1016/j.cll.2018.10.005

17. Adom D, Rowan C, Adeniyan T, Yang J, Paczesny S. Biomarkers for Allogeneic HCT Outcomes. Front Immunol (2020) 11(April):1-13. doi: 10.3389/fimmu. 2020.00673

18. Ferrara J, Levine J, Reddy P, Holler E. Graft-versus-host disease. Lancet (2009) 373(9674):1550-61. doi: 10.1016/S0140-6736(09)60237-3

19. Bäuerlein CA, Riedel SS, Baker J, Brede C, Jordán Garotte AL, Chopra M, et al. A diagnostic window for the treatment of acute graft-versus-host disease prior to visible clinical symptoms in a murine model. BMC Med (2013) 11(1):1-9. doi: 10.1186/1741-7015-11-134

20. Podgorny PJ, Liu Y, Dharmani-Khan P, Pratt LM, Jamani K, Luider J, et al. Immune cell subset counts associated with graft-versus-host disease. Biol Blood Marrow Transplant (2014) 20(4):450-62. doi: 10.1016/ j.bbmt.2014.01.002

21. Wysocki CA, Panoskaltsis-Mortari A, Blazar BR, Serody JS. Leukocyte migration and graft-versus-host disease. Blood (2005) 105(11):4191-9. doi: 10.1182/blood-2004-12-4726

22. Li W, Liu L, Gomez A, Zhang J, Ramadan A, Zhang Q, et al. Proteomics analysis reveals a Th17-prone cell population in presymptomatic graft-versushost disease. JCI Insight (2016) 1(6):e86660. doi: 10.1172/jci.insight.86660

23. Cooke K, Kobzik L, Martin T, Brewer J, Delmonte JJ, Crawford J, et al. An experimental model of idiopathic pneumonia syndrome after bone marrow transplantation: 1. The roles of minor $\mathrm{H}$ antigens and endotoxin. Blood (1996) 88(8):3230-9. doi: 10.1182/blood.V88.8.3230.bloodjournal8883230
24. Lerner K, Kao G, Storb R, Buckner C, Clift R, Thomas E. Histopathology of graft-vs.-host reaction (GvHR) in human recipients of marrow from HL-A -matched sibling donors. Transplant Proc (1974) 6:367-71.

25. Petrovic A, Alpdogan O, Willis LM, Eng JM, Greenberg AS, Kappel BJ, et al. LPAM ( $\alpha 4 \beta 7$ integrin) is an important homing integrin on alloreactive T cells in the development of intestinal graft-versus-host disease. Blood (2004) 103 (4):1542-7. doi: 10.1182/blood-2003-03-0957

26. Waldman E, Lu S, Hubbard V, Kochman AA, Eng JM, Terwey TH, et al. Absence of $\beta 7$ integrin results in less graft-versus-host disease because of decreased homing of alloreactive T cells to intestine. Blood (2006) 107 (4):1703-11. doi: 10.1182/blood-2005-08-3445

27. Beilhack A, Schulz S, Baker J, Beilhack GF, Nishimura R, Baker EM, et al. Prevention of acute graft-versus-host disease by blocking T-cell entry to secondary lymphoid organs. Blood (2008) 111(5):2919-28. doi: 10.1182/blood-2007-09-112789

28. Chen Y, Cutler C. Biomarkers for acute GVHD: can we predict the unpredictable? Bone Marrow Transplant (2012) 48(6):755-60. doi: 10.1038/ bmt.2012.143

29. Chen Y, Kim H, McDonough S, Odze RD, Yao X, Lazo-Kallanian S, et al. UpRegulation of alpha4beta7 integrin on peripheral $\mathrm{T}$ cell subsets correlates with the development of acute intestinal graft-versus-host disease following allogeneic stem cell transplantation. Biol Blood Marrow Transplant (2013) 48(4):1066-76. doi: 10.1016/j.bbmt.2009.05.003

30. Engelhardt BG, Sengsayadeth SM, Jagasia M, Savani BN, Kassim AA, Lu P, et al. Tissue-specific regulatory $\mathrm{T}$ cells: Biomarker for acute graft-vs-host disease and survival. Exp Hematol (2012) 40(12):974-82. doi: 10.1016/ j.exphem.2012.08.002

31. Fuchs F, Schillinger D, Atreya R, Hirschmann S, Fischer S, Neufert C, et al. Clinical response to vedolizumab in ulcerative colitis patients is associated with changes in integrin expression profiles. Front Immunol (2017) 8(764):112. doi: 10.3389 /fimmu.2017.00764

32. Asaduzzaman M, Mihaescu A, Wang Y, Sato T, Thorlacius H. P-Selectin and P-Selectin Glycoprotein Ligand 1 Mediate Rolling of Activated CD8+ T Cells in Inflamed Colonic Venules. J Invest Med (2009) 57(7):765-8. doi: 10.2310/ JIM.0b013e3181b918fb

33. Ali N, Flutter B, Sanchez Rodriguez R, Sharif-Paghaleh E, Barber LD, Lombardi G, et al. Xenogeneic Graft-versus-Host-Disease in NOD-scid IL2R $\gamma$ null Mice Display a T-Effector Memory Phenotype. PloS One (2012) 7 (8):1-10. doi: 10.1371/journal.pone.0044219

34. Eom H, Rubio M, Means T, Luster A, Sykes M. T-cell P/E-selectin ligand $\alpha(1,3)$ fucosylation is not required for graft-vs-host disease induction. Exp Hematol (2005) 33(12):1564-73. doi: 10.1016/j.exphem.2005.08.003

35. Khandelwal P, Chaturvedi V, Owsley E, Davies SM, Marsh RA. CD38 Bright CD8+ TEM Cells Detected Prior to Acute Gvhd are Activated, Cytotoxic, Proliferating, Trafficking Cells Which are Not Viral Specific. Biol Blood Marrow Transplant (2017) 23(3):137. doi: 10.1016/j.bbmt.2017.01.057

36. Khandelwal P, Chaturvedi V, Owsley E, Lane A, Heyenbruch D, Lutzko CM, et al. CD38brightCD8+ T Cells Associated with the Development of Acute GVHD Are Activated, Proliferating, and Cytotoxic Trafficking Cells. Biol Blood Marrow Transplant (2020) 26(1):1-6. doi: 10.1016/j.bbmt.2019.08.008

37. Zeiser R, Blazar B. Preclinical models of acute and chronic graft-versus-host disease: how predictive are they for a successful clinical translation? Blood (2016) 127(25):3117-27. doi: 10.1182/blood-2016-02-699082

38. Bouazzaoui A, Spacenko E, Mueller G. Steroid treatment alters adhesion molecule and chemokine expression in experimental acute graft-vs.-host disease of the intestinal tract. Exp Hematol (2011) 39(2):238-41. doi: 10.1016/j.exphem.2010.11.006

39. Munoz L, Holgado B, Martinez A, Rodriguez-Frade J, Mellado M. Chemokine receptor oligomerization: a further step toward chemokine function. Immunol Lett (2012) 145(1-2):23-9. doi: 10.1016/j.imlet.2012.04.012

40. Eyrich M, Burger G, Marquardt K, Budach W, Schilbach K, Niethammer D, et al. Sequential expression of adhesion and costimulatory molecules in graftversus-host disease target organs after murine bone marrow transplantation across minor histocompatibility antigen barriers. Biol Blood Marrow Transplant (2005) 11(5):371-82. doi: 10.1016/j.bbmt.2005.02.002

41. Chakraverty R, Côté D, Buchli J, Cotter P, Hsu R, Zhao G, et al. An inflammatory checkpoint regulates recruitment of graft-versus-host reactive T cells to peripheral tissues. J Exp Med (2006) 203(8):2021-31. doi: 10.1084/ jem.20060376 
42. Reshef R, Luger S, Hexner E, Loren AW, Frey NV, Nasta SD, et al. Blockade of lymphocyte chemotaxis in visceral graft-versus-host disease. $N$ Engl J Med (2012) 367(2):135-45. doi: 10.1056/NEJMoa1201248

43. Reshef R, Ganetsky A, Acosta EP, Blauser R, Crisalli L, McGraw J, et al. Extended CCR5 Blockade for Graft-versus-Host Disease Prophylaxis Improves Outcomes of Reduced-Intensity Unrelated Donor Hematopoietic Cell Transplantation: A Phase II Clinical Trial. Biol Blood Marrow Transplant (2019) 25(3):515-21. doi: 10.1016/j.bbmt.2018.09.034

44. Murai M, Yoneyama H, Harada A, Yi Z, Verstergaard C, Guo B, et al. Active participation of CCR $5+\mathrm{CD} 8+\mathrm{T}$ lymphocytes in the pathogenesis of liver injury in graft-versus-host disease. J Clin Invest (1999) 104(1):49-57. doi: 10.1172/JCI6642

45. Murai M, Yoneyama H, Ezaki T, Suematsu M, Terashima Y, Harada A, et al. Peyer's patch is the essential site in initiating murine acute and lethal graftversus-host reaction. Nat Immunol (2003) 4(2):154-60. doi: 10.1038/ni879

46. Paczesny S, Krijanovski O, Braun T, Choi SW, Glouthier SG, Kuick R, et al. A biomarker panel for acute graft-versus-host disease. Blood (2009) 113(2):2738. doi: 10.1182/blood-2008-07-167098

47. Srinagesh H, Özbek U, Kapoor U, Ayuk F, Aziz M, Ben-David K, et al. The MAGIC algorithm probability is a validated response biomarker of treatment of acute graft-versus-host disease. Blood $A d v$ (2019) 3(23):4034-42. doi: 10.1182/bloodadvances.2019000791

48. Weissinger E, Metzger J, Dobbelstein C, Wolff D, Schleuning M, Kuzmina Z, et al. Proteomic peptide profiling for preemptive diagnosis of acute graftversus-host disease after allogeneic stem cell transplantation. Leukemia (2014) 28(4):842-52. doi: 10.1038/leu.2013.210

Conflict of Interest: The authors declare that the research was conducted in the absence of any commercial or financial relationships that could be construed as a potential conflict of interest.

Copyright (๑ 2021 Bäuerlein, Qureischi, Mokhtari, Tabares, Brede, Jordán Garrote, Riedel, Chopra, Reu, Mottok, Arellano-Viera, Graf, Kurzwart, Schmiedgen, Einsele, Wölfl, Schlegel and Beilhack. This is an open-access article distributed under the terms of the Creative Commons Attribution License (CC BY). The use, distribution or reproduction in other forums is permitted, provided the original author(s) and the copyright owner(s) are credited and that the original publication in this journal is cited, in accordance with accepted academic practice. No use, distribution or reproduction is permitted which does not comply with these terms. 